\author{
Military Technical College \\ Kobry El-Kobbah, \\ Cairo, Egypt.
}

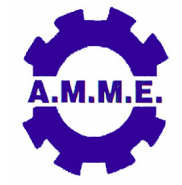
$15^{\text {th }}$ International Conference on Applied Mechanics and Mechanical Engineering.

\title{
ANALYTIC SOLUTIONS OF INHOMOGENEOUS AND NONLINEAR PROBLEMS OF HEAT CONDUCTION THEORY FOR A LAYER
}

\author{
A. N. Tyurehodzhayev* and G. A. Karibayeva**
}

\begin{abstract}
The article consider inhomogeneous and non-linear heat problems by applying the method of partial discretization of nonlinear differential equations, derived by Professor $\mathrm{A}$. $\mathrm{N}$. Tyurehodzhayev and methods of mathematical physics connected with the use of integral Laplace transforms. The aim of work is to obtain analytical solutions of boundary-value problems of inhomogeneous and nonlinear heat conduction by applying the method of partial discretization of nonlinear differential equations, establishing of regularity of heat distribution in the layer, which describe the differential equations in partial derivatives of parabolic type with variable mechanical and thermal characteristics, in some cases dependent on the unknown function itself. This paper addresses the following objectives: 1) Inhomogeneous problem of heat conduction theory with different dependences of heat conduction coefficient, heat capacity and medium density. 2) Non-linear problem of heat conduction with variable of heat capacity, density and heat conduction coefficient, which depends on the unknown function itself.
\end{abstract}

In regards to the problems of heat-conduction fundamental works are those of A.V. Lykov [45], L. M. Belyaev and A. A. Ryadno [6-7], V. S. Zarubin [8]. Among foreign authors, who have been solving the problem of this kind, we note the work of G. Carslaw and D. Jaeger [9], L.A. Kozdoba [10-11], and other heat-conduction investigators.

Work of L. I. Kudryashev and N. L. Menshih [12], a series of articles [13-15], etc. are devoted to the nonlinear problems of heat-conduction and methods of their solving. Application of the method of local potential in the heat conduction problems is described in the works of $P$. Glansdorff and I. Prigogine [16] R. Schechter [17].

In this article for the first time three were obtained analytical solutions of new problems of heat conduction with almost random variables and nonlinear thermal characteristics in the layer using the method of partial discretization of nonlinear differential equations of Professor A. Tyurehodzhaev by two variables, along with the integral Laplace transform.

\section{KEY WORDS}

Heat conduction, Nonlinear equations, Discretization, Variable characteristics, Temperature

\footnotetext{
* Professor, Department of Applied Mechanics and Principles of Machinery Engineering, the Kazakh National Technical University named after K.I. Satpayev, Almaty, Kazakhstan.

** Senior Lecturer, Department of Higher Mathematics and Physics, Almaty Technological University, Almaty, Kazakhstan.
} 


\section{NOTATION SYSTEM}

$\begin{array}{ll}c(x) & \text { Specific heat capacity. } \\ u(x, t) & \text { Temperature. } \\ f(x, t) & \text { Heat inflow. } \\ \bar{f}(p) & \text { Image of function. } \\ f(t), k(x) & \text { heat conduction coefficients. } \\ p & \text { Complex parameter. } \\ H\left(x-x_{k}\right) & \text { Heaviside unit function. } \\ \rho(x) & \text { Density. } \\ \delta\left(x-x_{k}\right) & \text { Dirac delta function. }\end{array}$

\section{ANALYTICAL SOLUTION OF THE INHOMOGENEOUS HEAT CONDUCTION EQUATION WITH HEAT INFLOW FOR THE LAYER}

We consider the inhomogeneous nonlinear problem on heat conduction for a variable coefficient of heat conduction, heat capacity and density of the object under consideration. By applying the method of partial discretization of nonlinear differential equations [1], we have obtained a solution of problem for the general case of the laws of change of thermophysical properties.

The differential equation of heat conduction in this case has the following form:

$$
\frac{\partial}{\partial x}\left(k(x) \frac{\partial u(x, t)}{\partial x}\right)+f(x, t)=\rho(x) c(x) \frac{\partial u(x, t)}{\partial t},
$$

Let's assume the following initial and boundary conditions are:

$$
\begin{aligned}
& u(x, 0)=\varphi(x), \\
& u(0, t)=\mu_{1}(t), \\
& u(l, t)=\mu_{2}(t) .
\end{aligned}
$$

Using the integral Laplace transform [2]

$$
\int_{0}^{\infty} e^{-p t} f(t) d t=\bar{f}(p)
$$

With the help of Laplace transform, the equation (1) can be written in the images

$$
\frac{d}{d x}\left(k(x) \frac{d \bar{u}(x, p)}{d x}\right)+\bar{f}(x, p)=\rho(x) c(x)[p \cdot \bar{u}(x, p)-\varphi(x)] .
$$

By applying the Laplace transform to the initial and boundary conditions (2), we have 


$$
\begin{aligned}
& \bar{u}(x, 0)=\frac{\varphi(x)}{p}, \\
& \bar{u}(0, p)=\bar{\mu}_{1}(p), \\
& \bar{u}(l, p)=\bar{\mu}_{2}(p) .
\end{aligned}
$$

Integration (3) gives:

$$
k(x) \frac{d \bar{u}(x, p)}{d x}=\int \rho(x) c(x)(p \bar{u}(x, p)-\varphi(x)) d x-\int \bar{f}(x, p) d x+A(p) .
$$

By discretizing the first integration element of the right part, we obtain:

$$
\begin{gathered}
\bar{u}(x, p)=\frac{1}{2} \int\left(\sum ( x _ { k } + x _ { k + 1 } ) \left[\rho\left(x_{k}\right) c\left(x_{k}\right)\left(p \bar{u}\left(x_{k}, p\right)-\varphi\left(x_{k}\right)\right) \frac{1}{k(x)} H\left(x-x_{k}\right)-\right.\right. \\
\left.\left.-\rho\left(x_{k+1}\right) c\left(x_{k+1}\right)\left(p \bar{u}\left(x_{k+1}, p\right)-\varphi\left(x_{k+1}\right)\right) \frac{1}{k(x)} H\left(x-x_{k+1}\right)\right]\right) d x- \\
-\int \frac{1}{k(x)} \int \bar{f}(x, p) d x d x+A(p) \int \frac{1}{k(x)} d x+B(p),
\end{gathered}
$$

For brevity, assume that

$$
\bar{F}(x, p)=\int \frac{1}{k(x)} \int \bar{f}(x, p) d x d x, \quad K(x)=\int \frac{1}{k(x)} d x .
$$

Therefore,

$$
\begin{gathered}
\bar{u}(x, p)=\frac{1}{2} \sum\left(x_{k}+x_{k+1}\right)\left[\rho\left(x_{k}\right) c\left(x_{k}\right)\left(p \bar{u}\left(x_{k}, p\right)-\varphi\left(x_{k}\right)\right)\left(K(x)-K\left(x_{k}\right)\right) H\left(x-x_{k}\right)-\right. \\
\left.\left.-\rho\left(x_{k+1}\right) c\left(x_{k+1}\right)\left(p \bar{u}\left(x_{k+1}, p\right)-\varphi\left(x_{k+1}\right)\right)\left(K(x)-K\left(x_{k+1}\right)\right) H\left(x-x_{k+1}\right)\right]\right] d x- \\
-\bar{F}(x, p)+A(p) K(x)+B(p) .
\end{gathered}
$$

Introducing the notation

$$
Z\left(x, x_{k}\right)=\rho\left(x_{k}\right) c\left(x_{k}\right)\left(K(x)-K\left(x_{k}\right)\right) H\left(x-x_{k}\right) .
$$

By using the boundary conditions (4), we obtain:

$$
\begin{gathered}
\bar{u}(x, p)=\frac{1}{2} \sum\left(x_{k}+x_{k+1}\right)\left[Z\left(x, x_{k}\right)\left(p \bar{u}\left(x_{k}, p\right)-\varphi\left(x_{k}\right)\right)-Z\left(x, x_{k+1}\right) \times\right. \\
\left.\times\left(p \bar{u}\left(x_{k+1}, p\right)-\varphi\left(x_{k+1}\right)\right)\right]-D(x) \frac{1}{2} \sum\left(x_{k}+x_{k+1}\right)\left[Z\left(l, x_{k}\right)\left(p \bar{u}\left(x_{k}, p\right)-\varphi\left(x_{k}\right)\right)-\right.
\end{gathered}
$$




$$
\left.-Z\left(l, x_{k+1}\right)\left(p \bar{u}\left(x_{k+1}, p\right)-\varphi\left(x_{k+1}\right)\right)\right]+\bar{\Phi}(x, p),
$$

where

$$
\begin{gathered}
D(x)=\frac{K(x)-K(0)}{K(l)-K(0)} \\
\bar{\Phi}(x, p)=-\bar{F}(x, p)+\bar{F}(0, p)+\bar{\mu}_{1}(p)+\frac{K(x)-K(0)}{K(l)-K(0)} \cdot\left[\bar{\mu}_{2}(p)-\bar{\mu}_{1}(p)+\bar{F}(l, p)-\bar{F}(0, p)\right] .
\end{gathered}
$$

By applying inverse Laplace transform, we obtain the original $u(x, t)$

$$
\begin{gathered}
u(x, t)=\frac{1}{2} \sum\left(x_{k}+x_{k+1}\right)\left[\left(Z\left(x, x_{k}\right)-D(x) Z\left(l, x_{k}\right)\right) \frac{d u\left(x_{k}, t\right)}{d t}-\right. \\
\left.-\left(Z\left(x, x_{k+1}\right)-D(x) Z\left(l, x_{k+1}\right)\right) \frac{d u\left(x_{k+1}, t\right)}{d t}\right]+\Phi(x, t)
\end{gathered}
$$

Let's define a formula for $u\left(x_{k}, t\right)$ in points $x_{k}$

At $k=1, x_{1}=0$

$$
u\left(x_{1}, t_{m}\right)=\mu_{1}\left(t_{m}\right)
$$

At $k=2$

$$
\frac{d u\left(x_{2}, t\right)}{d t}=\frac{1}{a_{2}}\left(\frac{1}{2}\left(x_{1}+x_{2}\right) \frac{d u\left(x_{1}, t\right)}{d t}\left(Z\left(x_{2}, x_{1}\right)-D\left(x_{2}\right) Z\left(l, x_{1}\right)\right)+\Phi\left(x_{2}, t\right)\right)-\frac{1}{a_{2}} u\left(x_{2}, t\right),
$$

where

$$
a_{2}=\frac{1}{2}\left(x_{3}-x_{1}\right) D\left(x_{2}\right) Z\left(l, x_{2}\right) \text {. }
$$

By discretizing the last element, we have

$$
\begin{gathered}
\frac{d u\left(x_{2}, t\right)}{d t}=\frac{1}{a_{2}}\left(\frac{1}{2}\left(x_{1}+x_{2}\right) \frac{d u\left(x_{1}, t\right)}{d t}\left(Z\left(x_{2}, x_{1}\right)-D\left(x_{2}\right) Z\left(l, x_{1}\right)\right)+\Phi\left(x_{2}, t\right)\right)- \\
-\frac{1}{2 a_{2}} \sum\left(t_{m}+t_{m+1}\right)\left[u\left(x_{2}, t_{m}\right) \delta\left(t-t_{m}\right)-u\left(x_{2}, t_{m+1}\right) \delta\left(t-t_{m+1}\right)\right] .
\end{gathered}
$$

After integration

$$
\begin{aligned}
& u\left(x_{2}, t\right)=\frac{1}{a_{2}}\left(\frac{1}{2}\left(x_{1}+x_{2}\right) u\left(x_{1}, t\right)\left(Z\left(x_{2}, x_{1}\right)-D\left(x_{2}\right) Z\left(l, x_{1}\right)\right)+\Phi 1\left(x_{2}, t\right)\right)- \\
& -\frac{1}{2 a_{2}} \sum\left(t_{m}+t_{m+1}\right)\left[u\left(x_{2}, t_{m}\right) H\left(t-t_{m}\right)-u\left(x_{2}, t_{m+1}\right) H\left(t-t_{m+1}\right)\right]+C_{2},
\end{aligned}
$$


where

$$
\Phi 1\left(x_{2}, t\right)=\int \Phi\left(x_{2}, t\right) d t
$$

Considering (1), we have

$$
\begin{gathered}
u\left(x_{2}, t\right)=\frac{1}{a_{2}}\left(\frac{1}{2}\left(x_{1}+x_{2}\right) u\left(x_{1}, t\right)\left(Z\left(x_{2}, x_{1}\right)-D\left(x_{2}\right) Z\left(l, x_{1}\right)\right)+\Phi 1\left(x_{2}, t\right)\right)- \\
-\frac{1}{2 a_{2}} \sum\left(t_{m}+t_{m+1}\right)\left[u\left(x_{2}, t_{m}\right) H\left(t-t_{m}\right)-u\left(x_{2}, t_{m+1}\right) H\left(t-t_{m+1}\right)\right]+\varphi\left(x_{2}\right)- \\
-\frac{1}{a_{2}}\left(\frac{1}{2}\left(x_{1}+x_{2}\right) \varphi\left(x_{1}\right)\left(Z\left(x_{2}, x_{1}\right)-D\left(x_{2}\right) Z\left(l, x_{1}\right)\right)+\Phi 1\left(x_{2}, 0\right)\right)+\frac{1}{2 a_{2}}\left(t_{1}+t_{2}\right) \varphi\left(x_{2}\right) .
\end{gathered}
$$

Let's define the formulas $u\left(x_{2}, t_{m}\right)$ in points $t_{m}$

$$
\begin{gathered}
m=1, \begin{array}{c}
u\left(x_{2}, t_{1}\right)=\varphi\left(x_{2}\right) \\
m=2
\end{array} \\
u\left(x_{2}, t_{2}\right)=\frac{1}{1+\frac{1}{2 a_{2}}\left(t_{3}-t_{1}\right)}\left\{\frac { 1 } { a _ { 2 } } \left(\frac{1}{2}\left(x_{1}+x_{2}\right) u\left(x_{1}, t_{2}\right)\left(Z\left(x_{2}, x_{1}\right)-D\left(x_{2}\right) Z\left(l, x_{1}\right)\right)+\right.\right. \\
\left.+\Phi 1\left(x_{2}, t_{2}\right)\right)-\frac{1}{2 a_{2}}\left(t_{1}+t_{2}\right) u\left(x_{2}, t_{1}\right)+\varphi\left(x_{2}\right)- \\
\left.-\frac{1}{a_{2}}\left(\frac{1}{2}\left(x_{1}+x_{2}\right) \varphi\left(x_{1}\right)\left(Z\left(x_{2}, x_{1}\right)-D\left(x_{2}\right) Z\left(l, x_{1}\right)\right)+\Phi 1\left(x_{2}, 0\right)\right)+\frac{1}{2 a_{2}}\left(t_{1}+t_{2}\right) \varphi\left(x_{2}\right)\right\} \\
u\left(x_{2}, t_{m}\right)=\frac{m=\overline{3, s}}{1-\frac{1}{2 a_{2}}\left(t_{m+1}-t_{m-1}\right)}\left\{\frac { 1 } { a _ { 2 } } \left(\frac{1}{2}\left(x_{1}+x_{2}\right) u\left(x_{1}, t_{m}\right)\left(Z\left(x_{2}, x_{1}\right)-D\left(x_{2}\right) Z\left(l, x_{1}\right)\right)+\right.\right. \\
\left.+\Phi 1\left(x_{2}, t_{m}\right)\right)-\frac{1}{2 a_{2}}\left[\left(t_{m-1}+t_{m}\right) u\left(x_{2}, t_{m-1}\right)+\sum_{j=1}^{m-2}\left(t_{j}+t_{j+1}\right)\left[u\left(x_{2}, t_{j}\right)-u\left(x_{2}, t_{j+1}\right)\right]+\varphi\left(x_{2}\right)-\right. \\
\left.-\frac{1}{a_{2}}\left(\frac{1}{2}\left(x_{1}+x_{2}\right) \varphi\left(x_{1}\right)\left(Z\left(x_{2}, x_{1}\right)-D\left(x_{2}\right) Z\left(l, x_{1}\right)\right)+\Phi 1\left(x_{2}, 0\right)\right)+\frac{1}{2 a_{2}}\left(t_{1}+t_{2}\right) \varphi\left(x_{2}\right)\right\}
\end{gathered}
$$

$$
\begin{gathered}
\text { At } k=\overline{3, n}, \\
\frac{d u\left(x_{k}, t\right)}{d t}=\frac{1}{a_{k}}\left\{\Phi\left(x_{k}, t\right)+\frac{1}{2}\left[( x _ { k - 1 } + x _ { k } ) \frac { d u ( x _ { k - 1 } , t ) } { d t } \left(Z\left(x_{k}, x_{k-1}\right)-\right.\right.\right.
\end{gathered}
$$$$
\left.-D\left(x_{k}\right) Z\left(l, x_{k-1}\right)\right)+\sum_{i=1}^{k-2}\left(x_{i}+x_{i+1}\right)\left[\frac{d u\left(x_{i}, t\right)}{d t}\left(Z\left(x_{k}, x_{i}\right)-D\left(x_{k}\right) Z\left(l, x_{i}\right)\right)-\frac{d u\left(x_{i+1}, t\right)}{d t} \times\right.
$$ 


$$
\left.\left.\left.\times\left(Z\left(x_{k}, x_{i+1}\right)-D\left(x_{k}\right) Z\left(l, x_{i+1}\right)\right)\right]\right]\right\}-\frac{1}{a_{k}} u\left(x_{k}, t\right) .
$$

where

$$
a_{k}=\frac{1}{2}\left(x_{k+1}-x_{k-1}\right) D\left(x_{k}\right) Z\left(l, x_{k}\right) \text {. }
$$

By discretizing the last element, we have

$$
\begin{gathered}
\frac{d u\left(x_{k}, t\right)}{d t}=\frac{1}{a_{k}}\left\{\Phi\left(x_{k}, t\right)+\frac{1}{2}\left[( x _ { k - 1 } + x _ { k } ) \frac { d u ( x _ { k - 1 } , t ) } { d t } \left(Z\left(x_{k}, x_{k-1}\right)-\right.\right.\right. \\
\left.-D\left(x_{k}\right) Z\left(l, x_{k-1}\right)\right)+\sum_{i=1}^{k-2}\left(x_{i}+x_{i+1}\right)\left[\frac{d u\left(x_{i}, t\right)}{d t}\left(Z\left(x_{k}, x_{i}\right)-D\left(x_{k}\right) Z\left(l, x_{i}\right)\right)-\frac{d u\left(x_{i+1}, t\right)}{d t} \times\right. \\
\left.\left.\left.\times\left(Z\left(x_{k}, x_{i+1}\right)-D\left(x_{k}\right) Z\left(l, x_{i+1}\right)\right)\right]\right]\right\}- \\
-\frac{1}{2 a_{k}} \sum\left(t_{m}+t_{m+1}\right)\left[u\left(x_{k}, t_{m}\right) \delta\left(t-t_{m}\right)-u\left(x_{k}, t_{m+1}\right) \delta\left(t-t_{m+1}\right)\right] .
\end{gathered}
$$

After integration considering the initial conditions (2)

$$
\begin{gathered}
u\left(x_{k}, t\right)=\frac{1}{a_{k}}\left\{\Phi 1\left(x_{k}, t\right)+\frac{1}{2}\left[( x _ { k - 1 } + x _ { k } ) u ( x _ { k - 1 } , t ) \left(Z\left(x_{k}, x_{k-1}\right)-\right.\right.\right. \\
\left.-D\left(x_{k}\right) Z\left(l, x_{k-1}\right)\right)+\sum_{i=1}^{k-2}\left(x_{i}+x_{i+1}\right)\left[u\left(x_{i}, t\right)\left(Z\left(x_{k}, x_{i}\right)-D\left(x_{k}\right) Z\left(l, x_{i}\right)\right)-u\left(x_{i+1}, t\right) \times\right. \\
\left.\left.\left.\times\left(Z\left(x_{k}, x_{i+1}\right)-D\left(x_{k}\right) Z\left(l, x_{i+1}\right)\right)\right]\right]\right\}- \\
-\frac{1}{2 a_{k}} \sum\left(t_{m}+t_{m+1}\right)\left[u\left(x_{k}, t_{m}\right) H\left(t-t_{m}\right)-u\left(x_{k}, t_{m+1}\right) H\left(t-t_{m+1}\right)\right]+ \\
+\varphi\left(x_{k}\right)-\frac{1}{a_{k}}\left\{\Phi 1\left(x_{k}, 0\right)+\frac{1}{2}\left[( x _ { k - 1 } + x _ { k } ) \varphi ( x _ { k - 1 } ) \left(Z\left(x_{k}, x_{k-1}\right)-\right.\right.\right. \\
\left.-D\left(x_{k}\right) Z\left(l, x_{k-1}\right)\right)+\sum_{i=1}^{k-2}\left(x_{i}+x_{i+1}\right)\left[\varphi\left(x_{i}\right)\left(Z\left(x_{k}, x_{i}\right)-D\left(x_{k}\right) Z\left(l, x_{i}\right)\right)-\varphi\left(x_{i+1}\right) \times\right. \\
\left.\left.\times\left(Z\left(x_{k}, x_{i+1}\right)-D\left(x_{k}\right) Z\left(l, x_{i+1}\right)\right)\right]\right\}+\frac{1}{2 a_{k}}\left(t_{1}+t_{2}\right) \varphi\left(x_{k}\right)
\end{gathered}
$$

where

$$
\Phi 1\left(x_{k}, t\right)=\int \Phi\left(x_{k}, t\right) d t
$$


Let's define the formulas $u\left(x_{2}, t_{m}\right) k=\overline{3, n}$ in points $t_{m}$ $m=1, t_{1}=0$

$m=2$

$$
u\left(x_{k}, t_{1}\right)=\varphi\left(x_{k}\right)
$$

$$
\begin{gathered}
u\left(x_{k}, t_{2}\right)=\frac{1}{1+\frac{1}{2 a_{k}}\left(t_{3}-t_{1}\right)}\left\langle\frac { 1 } { a _ { k } } \left\{\Phi 1\left(x_{k}, t_{2}\right)+\frac{1}{2}\left[( x _ { k - 1 } + x _ { k } ) u ( x _ { k - 1 } , t _ { 2 } ) \left(Z\left(x_{k}, x_{k-1}\right)-\right.\right.\right.\right. \\
\left.-D\left(x_{k}\right) Z\left(l, x_{k-1}\right)\right)+\sum_{i=1}^{k-2}\left(x_{i}+x_{i+1}\right)\left[u\left(x_{i}, t_{2}\right)\left(Z\left(x_{k}, x_{i}\right)-D\left(x_{k}\right) Z\left(l, x_{i}\right)\right)-u\left(x_{i+1}, t_{2}\right) \times\right. \\
\left.\left.\left.\times\left(Z\left(x_{k}, x_{i+1}\right)-D\left(x_{k}\right) Z\left(l, x_{i+1}\right)\right)\right]\right]\right\}-\frac{1}{2 a_{k}}\left(t_{1}+t_{2}\right) u\left(x_{k}, t_{1}\right)+ \\
+\varphi\left(x_{k}\right)-\frac{1}{a_{k}}\left\{\Phi 1\left(x_{k}, 0\right)+\frac{1}{2}\left[( x _ { k - 1 } + x _ { k } ) \varphi ( x _ { k - 1 } ) \left(Z\left(x_{k}, x_{k-1}\right)-\right.\right.\right. \\
\left.-D\left(x_{k}\right) Z\left(l, x_{k-1}\right)\right)+\sum_{i=1}^{k-2}\left(x_{i}+x_{i+1}\right)\left[\varphi\left(x_{i}\right)\left(Z\left(x_{k}, x_{i}\right)-D\left(x_{k}\right) Z\left(l, x_{i}\right)\right)-\varphi\left(x_{i+1}\right) \times\right. \\
\left.\left.\left.\left.\times\left(Z\left(x_{k}, x_{i+1}\right)-D\left(x_{k}\right) Z\left(l, x_{i+1}\right)\right)\right]\right]\right\}-\frac{1}{2 a_{k}}\left(t_{1}+t_{2}\right) \varphi\left(x_{k}\right)\right\rangle .
\end{gathered}
$$

$m=\overline{3, s}$

$$
\begin{gathered}
u\left(x_{k}, t_{m}\right)=\frac{1}{1+\frac{1}{2 a_{k}}\left(t_{m+1}-t_{m-1}\right)}\left\langle\frac { 1 } { a _ { k } } \left\{\Phi 1\left(x_{k}, t_{m}\right)+\frac{1}{2}\left[\left(x_{k-1}+x_{k}\right) u\left(x_{k-1}, t_{m}\right) \times\right.\right.\right. \\
\times\left(Z\left(x_{k}, x_{k-1}\right)-D\left(x_{k}\right) Z\left(l, x_{k-1}\right)\right)+\sum_{i=1}^{k-2}\left(x_{i}+x_{i+1}\right)\left[u\left(x_{i}, t_{m}\right)\left(Z\left(x_{k}, x_{i}\right)-D\left(x_{k}\right) Z\left(l, x_{i}\right)\right)-\right. \\
\left.\left.\left.-u\left(x_{i+1}, t_{m}\right)\left(Z\left(x_{k}, x_{i+1}\right)-D\left(x_{k}\right) Z\left(l, x_{i+1}\right)\right)\right]\right\}\right\}-\frac{1}{2 a_{k}}\left[\left(t_{m-1}+t_{m}\right) u\left(x_{k}, t_{m-1}\right)+\right. \\
\left.+\sum_{j=1}^{m-2}\left(t_{j}+t_{j+1}\right)\left[u\left(x_{k}, t_{j}\right)-u\left(x_{k}, t_{j+1}\right)\right]\right]+ \\
+\varphi\left(x_{k}\right)-\frac{1}{a_{k}}\left\{\Phi\left(x_{k}, 0\right)+\frac{1}{2}\left[( x _ { k - 1 } + x _ { k } ) \varphi ( x _ { k - 1 } ) \left(Z\left(x_{k}, x_{k-1}\right)-\right.\right.\right. \\
\left.-D\left(x_{k}\right) Z\left(l, x_{k-1}\right)\right)+\sum_{i=1}^{k-2}\left(x_{i}+x_{i+1}\right)\left[\varphi\left(x_{i}\right)\left(Z\left(x_{k}, x_{i}\right)-D\left(x_{k}\right) Z\left(l, x_{i}\right)\right)-\varphi\left(x_{i+1}\right) \times\right. \\
\left.\left.\left.\left.\times\left(Z\left(x_{k}, x_{i+1}\right)-D\left(x_{k}\right) Z\left(l, x_{i+1}\right)\right)\right]\right]\right\}-\frac{1}{2 a_{k}}\left(t_{1}+t_{2}\right) \varphi\left(x_{k}\right)\right\rangle .
\end{gathered}
$$




\section{Example}

For construction of a graph, let's consider a sample for the problem (1) - (2), under specific functions, for example, $k(x)=b_{3} e^{-a_{3} x}$ - heat conduction coefficient, $C(x)=c(x) \rho(x)=a_{1}+b_{1} x-$ volumetric heat capacity and $f(x, t)=\alpha_{3}+\frac{1}{2} \beta_{3} x t^{2}-$ heat inflow.

Then, equation (1) will be:

$$
\frac{\partial}{\partial x}\left(b_{3} e^{-a_{3} x} \frac{\partial u(x, t)}{\partial x}\right)+\left(\alpha_{3}+\frac{1}{2} \beta_{3} x t^{2}\right)=\left(a_{1}+b_{1} x\right) \frac{\partial u(x, t)}{\partial t} .
$$

Assume the following initial and boundary conditions

$$
\begin{aligned}
& u(x, 0)=\varphi(x)=a_{2}+b_{2} x^{2}, \\
& u(0, t)=\mu_{1}(t)=\alpha_{1}+e^{\beta_{1} t}, \\
& u(l, t)=\mu_{2}(t)=\alpha_{2}+\gamma_{2} t e^{-\beta_{2} t} .
\end{aligned}
$$

Thus, let's calculate the formulas $u\left(x_{k}, t_{m}\right) \quad k=\overline{1, n}, \quad m=\overline{1, s}$, at calculated data $\alpha_{1}=55^{\circ} \mathrm{C}, \quad \beta_{1}=0.3 \mathrm{~h}^{-1}, \quad \alpha_{2}=106^{\circ} \mathrm{C}, \quad \beta_{2}=0,4 \mathrm{~h}^{-1}, \quad \gamma_{2}=106 \frac{{ }^{\circ} \mathrm{C}}{\mathrm{h}}, \quad \alpha_{3}=3000 \mathrm{~J}$, $\beta_{3}=0,5 \frac{\mathrm{J}}{\mathrm{m} \cdot \mathrm{h}^{2}}, \quad \mathrm{a}_{1}=1,35 \cdot 10^{-6} \frac{\mathrm{J}}{\mathrm{m}^{3} \cdot{ }^{\circ} \mathrm{C}}, \quad b_{1}=0,5 \cdot 10^{-6} \frac{\mathrm{J}}{\mathrm{m}^{4} \cdot{ }^{\circ} \mathrm{C}}, \quad \mathrm{a}_{2}=56^{\circ} \mathrm{C}, \quad \mathrm{b}_{2}=0,5 \frac{{ }^{\circ} \mathrm{C}}{\mathrm{m}^{2}}$, $\mathrm{a}_{3}=0,07 \mathrm{~m}^{-1}, \mathrm{~b}_{3}=1,2 \frac{\mathrm{Watt}}{\mathrm{m} \cdot{ }^{\circ} \mathrm{C}}, \mathrm{l}=10 \mathrm{~m}$.

The solutions obtained are given in Figures $2-4$ at time interval $\mathrm{t}=0-15 \mathrm{~h}$.

\section{THE SOLUTION OF ONE NONLINEAR HEAT CONDUCTION EQUATION WITH NO HEAT SOURCE}

Let's consider the nonlinear heat conduction equation with variable thermophysical properties of the general form. Analytical solution of the problem is obtained by the method of partial discretization of nonlinear differential equations of Professor A. N. Tyurehodzhayev [3] and by means of Laplace transform.

The differential equation of heat conduction of the problem under consideration is:

$$
\frac{\partial}{\partial x}\left(k_{0} u(x, t) \frac{\partial u(x, t)}{\partial x}\right)=\rho(x) c(x) \frac{\partial u(x, t)}{\partial t},
$$

It follows 


$$
\frac{\partial^{2} u^{2}(x, t)}{\partial x^{2}}=\frac{2}{k_{0}} \rho(x) c(x) \frac{\partial u(x, t)}{\partial t}
$$

Assume the following initial and boundary conditions

$$
\begin{aligned}
& u(x, 0)=\varphi(x), \\
& u(0, t)=\mu_{1}(t), \\
& u(l, t)=\mu_{2}(t) .
\end{aligned}
$$

By applying Laplace transform to problem (21)-(22), we obtain:

$$
\begin{gathered}
\frac{d^{2}}{d x^{2}}\left[\overline{u^{2}}(x, p)\right]=\frac{2}{k_{0}} c(x) \rho(x)[p \bar{u}(x, p)-\varphi(x)], \\
\bar{u}(0, p)=\bar{\mu}_{1}(p), \\
\bar{u}(l, p)=\bar{\mu}_{2}(p) .
\end{gathered}
$$

Partial discretization of equation (24), gives:

$$
\begin{gathered}
\frac{d^{2}}{d x^{2}}\left[\overline{u^{2}}(x, p)\right]=\frac{1}{k_{0}} \sum\left(x_{k}+x_{k+1}\right)\left[c\left(x_{k}\right) \rho\left(x_{k}\right)\left[p \bar{u}\left(x_{k}, p\right)-\varphi\left(x_{k}\right)\right] \cdot \delta\left(x-x_{k}\right)-\right. \\
\left.-c\left(x_{k+1}\right) \rho\left(x_{k+1}\right)\left[p \bar{u}\left(x_{k+1}, p\right)-\varphi\left(x_{k+1}\right)\right] \cdot \delta\left(x-x_{k+1}\right)\right]
\end{gathered}
$$

By integration (26)

$$
\begin{gathered}
\overline{u^{2}}(x, p)=\frac{1}{k_{0}} \sum\left(x_{k}+x_{k+1}\right)\left[Y\left(x, x_{k}\right)\left[p \bar{u}\left(x_{k}, p\right)-\varphi\left(x_{k}\right)\right]-\right. \\
-Y\left(x, x_{k+1}\right)\left[p \bar{u}\left(x_{k+1}, p\right)-\varphi\left(x_{k+1}\right)\right]+A(p) x+B(p), \\
Y\left(x, x_{k}\right)=c\left(x_{k}\right) \rho\left(x_{k}\right)\left(x-x_{k}\right) H\left(x-x_{k}\right) .
\end{gathered}
$$

Considering boundary conditions (25)

$$
\begin{gathered}
\overline{u^{2}}(x, p)=\bar{M}(x, p)+\frac{1}{k_{0}} \sum\left(x_{k}+x_{k+1}\right)\left[Y\left(x, x_{k}\right)\left[p \bar{u}\left(x_{k}, p\right)-\varphi\left(x_{k}\right)\right]-\right. \\
\left.-Y\left(x, x_{k+1}\right)\left[p \bar{u}\left(x_{k+1}, p\right)-\varphi\left(x_{k+1}\right)\right]\right]-\frac{1}{k_{0}} \sum\left(x_{k}+x_{k+1}\right)\left[Y\left(l, x_{k}\right)\left[p \bar{u}\left(x_{k}, p\right)-\varphi\left(x_{k}\right)\right]-\right. \\
-Y\left(l, x_{k+1}\right)\left[p \bar{u}\left(x_{k+1}, p\right)-\varphi\left(x_{k+1}\right)\right]
\end{gathered}
$$

where 


$$
\bar{M}(x, p)=\overline{\mu_{1}^{2}}(p)+\frac{x}{l}\left(\overline{\mu_{2}^{2}}(p)-\overline{\mu_{1}^{2}}(p)\right)
$$

Inverse Laplace transform to equation (27)

$$
\begin{gathered}
u^{2}(x, t)=M(x, t)+\frac{1}{k_{0}} \sum\left(x_{k}+x_{k+1}\right)\left[Y\left(x, x_{k}\right) \frac{d u\left(x_{k}, t\right)}{d t}-Y\left(x, x_{k+1}\right) \frac{d u\left(x_{k+1}, t\right)}{d t}\right] \\
-\frac{1}{k_{0}} \sum\left(x_{k}+x_{k+1}\right)\left[Y\left(l, x_{k}\right) \frac{d u\left(x_{k}, t\right)}{d t}-Y\left(l, x_{+1}\right) \frac{d u\left(x_{k+1}, t\right)}{d t}\right]
\end{gathered}
$$

where

$$
M(x, t)=\mu_{1}^{2}(t)+\frac{x}{l}\left(\mu_{2}^{2}(t)-\mu_{1}^{2}(t)\right) .
$$

Definition of formula $u\left(x_{k}, t\right)$ in points $x_{k}$.

$$
\begin{aligned}
& k=1, x_{1}=0 . \\
& \qquad u\left(x_{1}, t\right)=\mu_{1}(t), \\
& k=2, \\
& \quad u^{2}\left(x_{2}, t\right)=M\left(x_{2}, t\right)+\frac{1}{k_{0}}\left(x_{1}+x_{2}\right)\left[Y\left(x_{2}, x_{1}\right)-Y\left(x_{2}, x_{1}\right)\right] \cdot \frac{d u\left(x_{1}, t\right)}{d t}-A_{2} \frac{d u\left(x_{2}, t\right)}{d t},
\end{aligned}
$$

where

$$
A_{2}=\frac{1}{k_{0}}\left(x_{3}-x_{1}\right) Y\left(l, x_{2}\right) \text {. }
$$

Consequently

$$
\frac{d u\left(x_{2}, t\right)}{d t}=\frac{1}{A_{2}} M\left(x_{2}, t\right)+\frac{1}{k_{0} A_{2}}\left(x_{1}+x_{2}\right)\left[Y\left(x_{2}, x_{1}\right)-Y\left(l, x_{1}\right)\right] \frac{d u\left(x_{1}, t\right)}{d t}-\frac{1}{A_{2}} u^{2}\left(x_{2}, t\right) \text {. }
$$

$k=\overline{3, n}$,

$$
\begin{gathered}
u^{2}\left(x_{k}, t\right)=M\left(x_{k}, t\right)+\frac{1}{k_{0}} \sum_{i=1}^{k-2}\left(x_{i}+x_{i+1}\right)\left[\left[Y\left(x_{k}, x_{i}\right)-Y\left(l, x_{i}\right)\right] \cdot \frac{d u\left(x_{i}, t\right)}{d t}-\right. \\
\left.-\left[Y\left(x_{k}, x_{i+1}\right)-Y\left(l, x_{i+1}\right)\right] \cdot \frac{d u\left(x_{i+1}, t\right)}{d t}\right]+\frac{1}{k_{0}}\left(x_{k-1}+x_{k}\right)\left[Y\left(x_{k}, x_{k-1}\right)-Y\left(l, x_{k-1}\right)\right] \times \\
\times \frac{d u\left(x_{k-1}, t\right)}{d t}-A_{k} \frac{d u\left(x_{k}, t\right)}{d t},
\end{gathered}
$$

where

$$
A_{k}=\frac{1}{k_{0}}\left(x_{k+1}-x_{k-1}\right) Y\left(l, x_{k}\right) \text {. }
$$

Consequently 


$$
\begin{gathered}
\frac{d u\left(x_{k}, t\right)}{d t}=\frac{1}{A_{k}} M\left(x_{k}, t\right)+\frac{1}{k_{0} A_{k}} \sum_{i=1}^{k-2}\left(x_{i}+x_{i+1}\right)\left[\left[Y\left(x_{k}, x_{i}\right)-Y\left(l, x_{i}\right)\right] \cdot \frac{d u\left(x_{i}, t\right)}{d t}-\right. \\
\left.-\left[Y\left(x_{k}, x_{i+1}\right)-Y\left(l, x_{i+1}\right)\right] \cdot \frac{d u\left(x_{i+1}, t\right)}{d t}\right]+\frac{1}{k_{0} A_{k}}\left(x_{k-1}+x_{k}\right)\left[Y\left(x_{k}, x_{k-1}\right)-Y\left(l, x_{k-1}\right)\right] \times \\
\times \frac{d u\left(x_{k-1}, t\right)}{d t}-\frac{1}{A_{k}} u^{2}\left(x_{k}, t\right)
\end{gathered}
$$

Integrating (31)-(32), and writing for $k=\overline{2, n}$

$$
u\left(x_{k}, t\right)=\frac{1}{A_{k}} M_{1}\left(x_{k}, t\right)+B_{k}(t)-\frac{1}{A_{k}} \int u^{2}\left(x_{k}, t\right) d t+C_{k},
$$

where

$$
\begin{gathered}
B_{2}(t)=\frac{1}{k_{0} A_{2}}\left(x_{1}+x_{2}\right)\left[Y\left(x_{2}, x_{1}\right)-Y\left(l, x_{1}\right)\right] u\left(x_{1}, t\right), \\
B_{k}(t)=\frac{1}{k_{0} A_{k}} \sum_{i=1}^{k-2}\left(x_{i}+x_{i+1}\right)\left[\left[Y\left(x_{k}, x_{i}\right)-Y\left(l, x_{i}\right)\right] \cdot u\left(x_{i}, t\right)-\left[Y\left(x_{k}, x_{i+1}\right)-Y\left(l, x_{i+1}\right)\right] \times\right. \\
\left.\times u\left(x_{i+1}, t\right)\right]+\frac{1}{k_{0} A_{k}}\left(x_{k-1}+x_{k}\right)\left[Y\left(x_{k}, x_{k-1}\right)-Y\left(l, x_{k-1}\right)\right] u\left(x_{k-1}, t\right), \\
M_{1}(x, t)=\int M(x, t) d t
\end{gathered}
$$

$C_{k}-$ The integration constants.

Partial discretization (33) per $t$, gives

$$
\begin{gathered}
u\left(x_{k}, t\right)=\frac{1}{A_{k}} M_{1}\left(x_{k}, t\right)+B_{k}(t)-\frac{1}{2 A_{k}} \sum\left(t_{m}+t_{m+1}\right)\left[u^{2}\left(x_{k}, t_{m}\right) H\left(t-t_{m}\right)-\right. \\
\left.-u^{2}\left(x_{k}, t_{m+1}\right) H\left(t-t_{m+1}\right)\right]+C_{k} .
\end{gathered}
$$

Considering the initial conditions (22)

$$
\begin{aligned}
& u\left(x_{k}, t\right)=\frac{1}{A_{k}}\left(M_{1}\left(x_{k}, t\right)-M_{1}\left(x_{k}, 0\right)\right)+B_{k}(t)-B_{k}(0)+\varphi\left(x_{k}\right)+\frac{1}{2 A_{k}}\left(t_{1}+t_{2}\right) \varphi^{2}\left(x_{k}\right)- \\
& -\frac{1}{2 A_{k}} \sum\left(t_{m}+t_{m+1}\right)\left[u^{2}\left(x_{k}, t_{m}\right) H\left(t-t_{m}\right)-u^{2}\left(x_{k}, t_{m+1}\right) H\left(t-t_{m+1}\right)\right]
\end{aligned}
$$

where

$$
B_{2}(0)=\frac{1}{k_{0} A_{2}}\left(x_{1}+x_{2}\right)\left[Y\left(x_{2}, x_{1}\right)-Y\left(l, x_{1}\right)\right] \varphi\left(x_{1}\right)
$$




$$
\begin{aligned}
B_{k}(0)= & \frac{1}{k_{0} A_{k}} \sum_{i=1}^{k-2}\left(x_{i}+x_{i+1}\right)\left[\left[Y\left(x_{k}, x_{i}\right)-Y\left(l, x_{i}\right)\right] \cdot \varphi\left(x_{i}\right)-\left[Y\left(x_{k}, x_{i+1}\right)-Y\left(l, x_{i+1}\right)\right] \times\right. \\
& \left.\times \varphi\left(x_{i+1}\right)\right]+\frac{1}{k_{0} A_{k}}\left(x_{k-1}+x_{k}\right)\left[Y\left(x_{k}, x_{k-1}\right)-Y\left(l, x_{k-1}\right)\right] \varphi\left(x_{k-1}\right) .
\end{aligned}
$$

Let's define the formula $u\left(x_{k}, t_{m}\right)$ in points $t_{m}$.

At $m=1, t_{1}=0$

At $m=2$.

$$
u\left(x_{k}, t_{1}\right)=\varphi\left(x_{k}\right)
$$

$$
\begin{gathered}
u\left(x_{k}, t_{2}\right)=\frac{1}{A_{k}}\left(M_{1}\left(x_{k}, t_{2}\right)-M_{1}\left(x_{k}, 0\right)\right)+B_{k}\left(t_{2}\right)-B_{k}(0)+\varphi\left(x_{k}\right)- \\
\frac{1}{2 A_{k}}\left(t_{3}-t_{1}\right) u^{2}\left(x_{k}, t_{2}\right) .
\end{gathered}
$$

At $m=\overline{3, s}$.

$$
\begin{gathered}
u\left(x_{k}, t_{m}\right)=\frac{1}{A_{k}}\left(M_{1}\left(x_{k}, t_{m}\right)-M_{1}\left(x_{k}, 0\right)\right)+B_{k}\left(t_{m}\right)-B_{k}(0)+\varphi\left(x_{k}\right)- \\
-\frac{1}{2 A_{k}} \sum_{j=1}^{m-2}\left(t_{j}+t_{j+1}\right)\left[u^{2}\left(x_{k}, t_{j}\right)-u^{2}\left(x_{k}, t_{j+1}\right)\right]-\frac{1}{2 A_{k}}\left(t_{m-1}+t_{m}\right) u^{2}\left(x_{k}, t_{m-1}\right)+ \\
+\frac{1}{2 A_{k}}\left(t_{1}+t_{2}\right) \varphi^{2}\left(x_{k}\right)-\frac{1}{2 A_{k}}\left(t_{m+1}-t_{m-1}\right) u^{2}\left(x_{k}, t_{m}\right) .
\end{gathered}
$$

This shows that the obtained algebraic quadratic equations with respect to $u\left(x_{k}, t_{m}\right)$ $(m=\overline{2, s})$, which in general form are written as

where

$$
a_{k, m} u^{2}\left(x_{k}, t_{m}\right)+u\left(x_{k}, t_{m}\right)+c_{k, m}=0
$$

$$
\begin{gathered}
a_{k, m}=\frac{1}{2 A_{k}}\left(t_{m+1}-t_{m-1}\right),(m=\overline{2, s}), \\
c_{k, 2}=-\frac{1}{A_{k}}\left(M_{1}\left(x_{k}, t_{2}\right)-M_{1}\left(x_{k}, 0\right)\right)-B_{k}\left(t_{2}\right)+B_{k}(0)-\varphi\left(x_{k}\right), \quad(m=2), \\
c_{k, m}=-\frac{1}{A_{k}}\left(M_{1}\left(x_{k}, t_{m}\right)-M_{1}\left(x_{k}, 0\right)\right)-B_{k}\left(t_{m}\right)+B_{k}(0)-\varphi\left(x_{k}\right)+ \\
+\frac{1}{2 A_{k}} \sum_{j=1}^{m-2}\left(t_{j}+t_{j+1}\right)\left[u^{2}\left(x_{k}, t_{j}\right)-u^{2}\left(x_{k}, t_{j}\right)\right]+\frac{1}{2 A_{k}}\left(t_{m-1}+t_{m}\right) u^{2}\left(x_{k}, t_{m-1}\right)- \\
-\frac{1}{2 A_{k}}\left(t_{1}+t_{2}\right) \varphi^{2}\left(x_{k}\right), \quad(m=\overline{3, s}) .
\end{gathered}
$$

Consequently, the solution of the problem takes the form 


$$
u_{1,2}\left(x_{k}, t_{m}\right)=\frac{-1 \pm \sqrt{1-4 a_{k, m} c_{k, m}}}{2 a_{k, m}}
$$

An analytical solution of heat conduction equation is obtained by heat conduction coefficient that is linearly dependent on temperature, and with variable specific heat capacity and density.

\section{Example}

For construction of graph, let's consider a sample for problem (22)-(23), under specific functions, e.g. $k(x, t)=k_{0} u(x, t)$ and $C(x)=c(x) \rho(x)=a_{1}+b_{1} x$.

Let's assume the following initial and boundary conditions

$$
\begin{aligned}
& u(x, 0)=\varphi(x)=a_{2}+b_{2} x^{2}, \\
& u(0, t)=\mu_{1}(t)=\alpha_{1}+10 e^{\beta_{1} t}, \\
& u(l, t)=\mu_{2}(t)=\alpha_{2}-\gamma_{2} t^{2} e^{-\beta_{2} t}
\end{aligned}
$$

The graph is constructed at calculated data $\mathrm{k}_{0}=0.08 \frac{\text { Watt }}{\mathrm{m} \cdot\left({ }^{\circ} \mathrm{C}\right)^{2}}, \quad \alpha_{1}=55^{\circ} \mathrm{C}$, $\beta_{1}=-0.4 \mathrm{~h}^{-1}, \quad \alpha_{2}=106^{\circ} \mathrm{C}, \quad \beta_{2}=0.57 \mathrm{~h}^{-1}, \quad \gamma_{2}=50 \frac{{ }^{\circ} \mathrm{C}}{\mathrm{h}}, \quad \mathrm{a}_{1}=1.35 \cdot 10^{-6} \frac{\mathrm{J}}{\mathrm{m}^{3} \cdot{ }^{\circ} \mathrm{C}}$, $\mathrm{b}_{1}=0.5 \cdot 10^{-6} \frac{\mathrm{J}}{\mathrm{m}^{4} \cdot{ }^{\circ} \mathrm{C}}, \mathrm{a}_{2}=65^{\circ} \mathrm{C}, \mathrm{b}_{2}=0,5 \frac{{ }^{\circ} \mathrm{C}}{\mathrm{m}^{2}}, \quad 1=10 \mathrm{~m}$.

\section{CONCLUSION}

We obtained an analytical solution of heat conduction by the coefficient of thermal conductivity linearly dependent on temperature, and with a variable specific heat and density, and built three-dimensional picture of the temperature distribution in the layer.

Results of the study can be used in establishing of inhomogeneous and nonlinear laws of heat propagation in layered soils with variable and discontinuous mechanical properties, in design of power engineering, aircraft, missile equipment and other industries under the influence of power and temperature.

\section{REFERENCES}

[1] A. N. Tyurehodzhayev and G. A. Karibayeva - The analytical solution of the heat conduction equation with variable thermophysical properties // The Herald of KazNTU. - Almaty, 2010. - No. 5. - p.p. 257-264. 
[2] Gustav Doetsch. Guide to practical application of Laplace transform and Ztransform. - Moscow: Nauka, 1971. - p. 288.

[3] A. N. Tyurehodzhayev and G. A. Karibayeva - The solution of one nonlinear heat conduction equation // The Herald of Academy of Sciences of RK. Almaty, 2010. - No. 5. - p.37- 43.

[4] A.V. Lykov. The theory of heat conduction. - M.: High School, 1967. - 599p.

[5] A.V. Lykov. Heat and mass transfer. Handbook. . 2nd publ rev. and add. Moscow: Energiya, 1978. - 478p.

[6] N. M. Belyaev, A. A. Ryadno. Methods of the theory of heat conduction. - M. Story. izdat., 1982. - 447p.

[7] N. M. Belyaev, V. I. Vavelin and A. A. Ryadno. Projection and difference methods in heat transfer and thermoelasticity. - Dnepropetrovsk: Dnepropetrovsk State University, 1982. - 104c.

[8] V. S. Zarubin. Engineering methods for solving heat conduction problems. Moscow: Energoizdat, 1983. - 328p.

[9] H. Carslaw, D. Jaeger. The thermal conductivity of solids. - Moscow: Nauka, 1964. - 487p.

[10] L. A. Kozdoba. Methods for solving nonlinear heat conduction problems. Moscow: Nauka, 1975. - 228p.

[11] L. A. Kozdoba Solutions of nonlinear heat conduction problems. - Kiev: Naukova Dumka, 1976. - 136p.

[12] L. I. Kudryashov and N. L. Menshih. Approximate solutions of nonlinear heat conduction problems. - Moscow: Mashinostroenie, 1979. - 232C.

[13] Yu. N. Akkuratov and V. N. Mikhailov. The method of boundary integral equations for solving nonlinear problems of heat conduction // Journal of computational mathematics and Math. physics. - 1980. - T.29, № 3. - P.656663.

[14] O. L. Kardyuk. Application of the method of least squares for solutions of stationary nonlinear heat conduction problems // Thermophysics and Heat Engineering. - 1977. num.33. - P.87-89.

[15] A. I. Uzdalev and E. I. Bryukhanova. Problem of heat conduction for doubly connected plate with the thermal temperature-dependent properties // Mechanics of Inhomogeneous Structures: Materials of the 1st All-Union conference. Lvov. - Kiev, 1986. - P. 260-265.

[16] Glansdorff $P$. Thermodynamic theory of structure, stability and fluctuations. M.: Mir, 1973. - 280p.

[17] J. Schechter. The variational method in engineering calculations - M.: Mir, 1971. - 292p. 


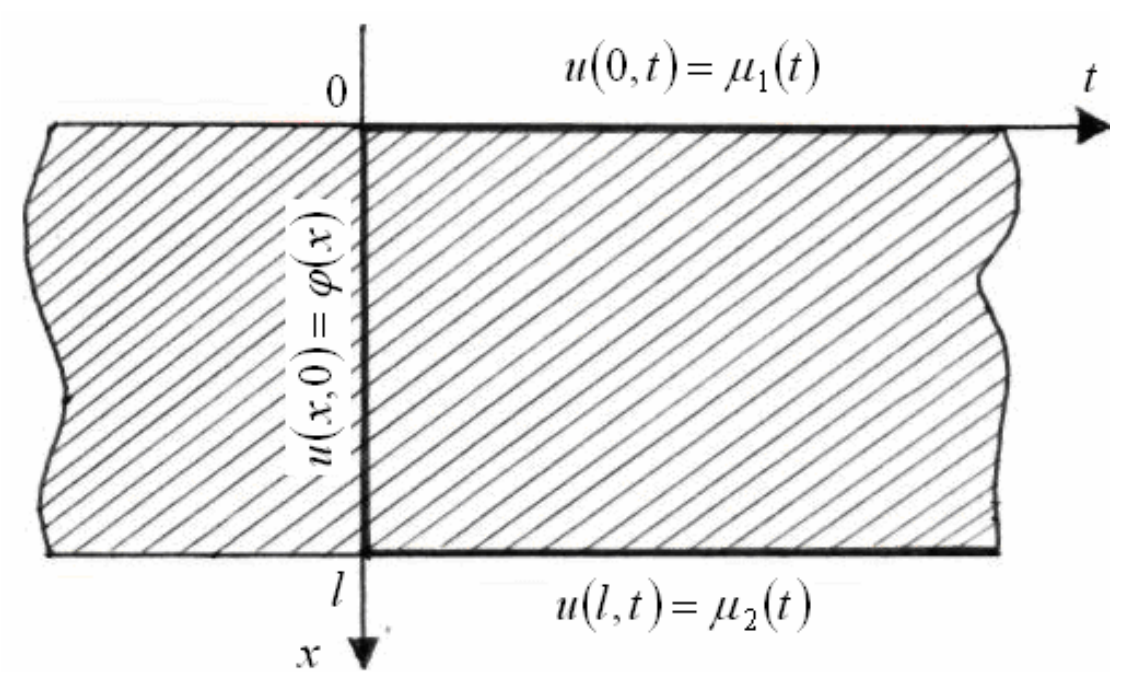

Figure1. The initial and boundary conditions for layer.

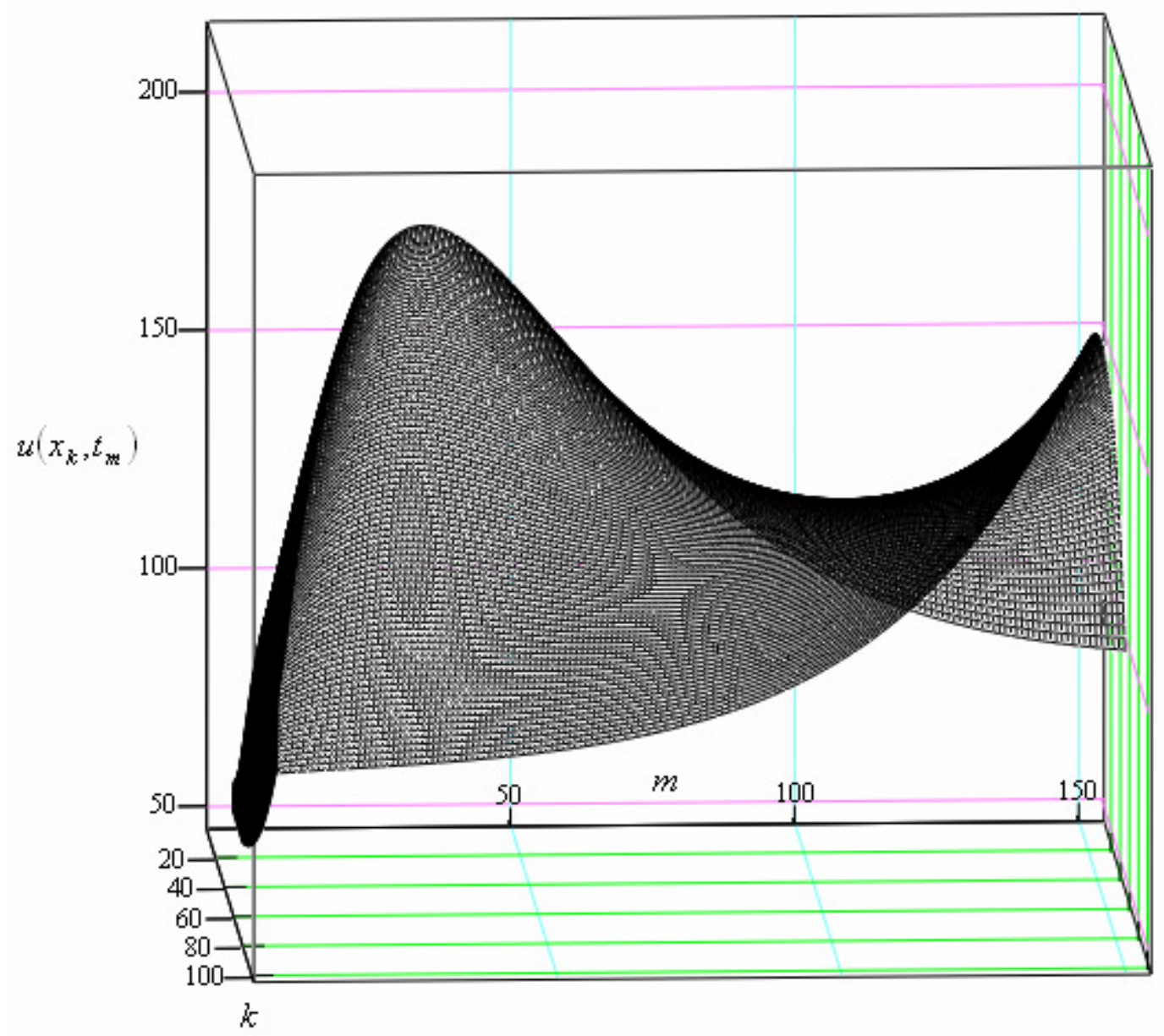

Figure 2. The graph of temperature change $u=u\left(x_{k}, t_{m}\right)$. 


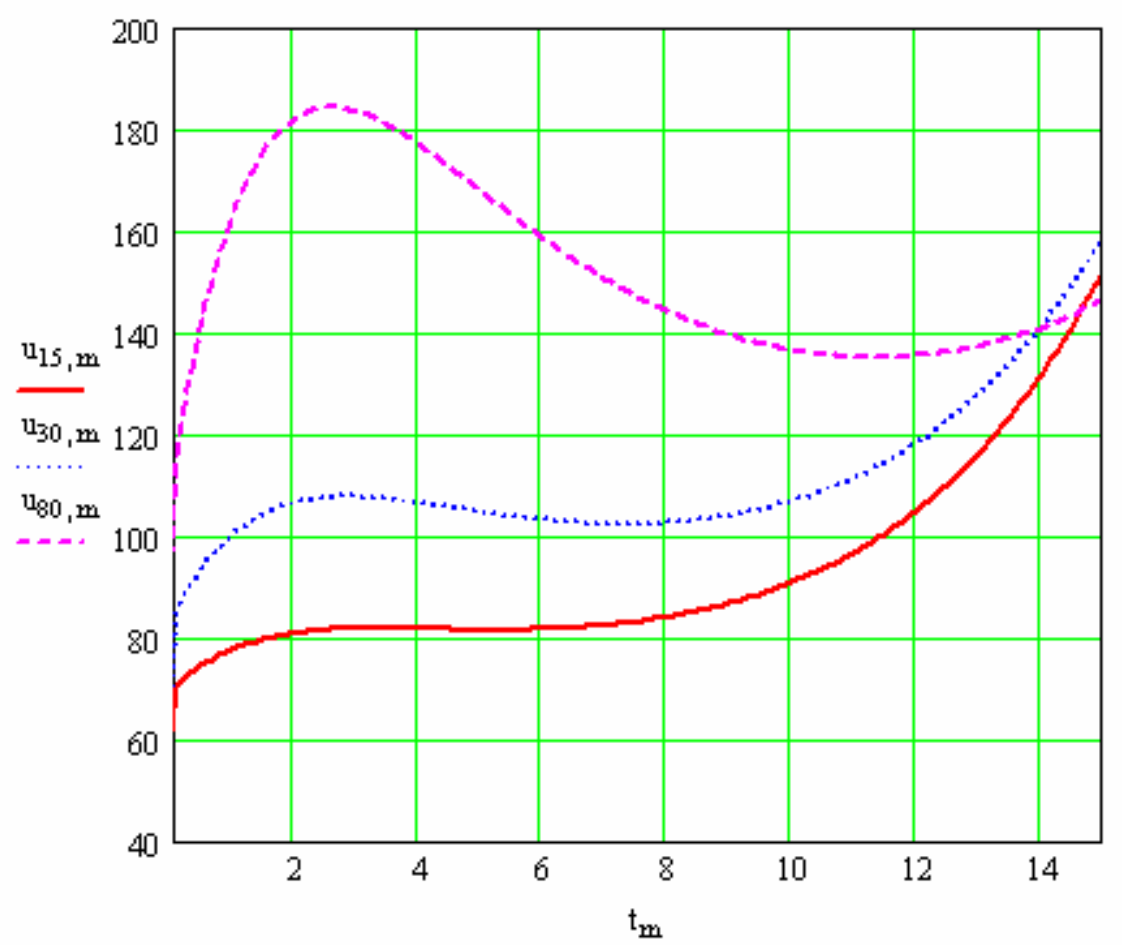

Figure 3. Dependence of temperature on time in layer sections $u\left(x_{15}, t_{m}\right), u\left(x_{30}, t_{m}\right), u\left(x_{80}, t_{m}\right)$.

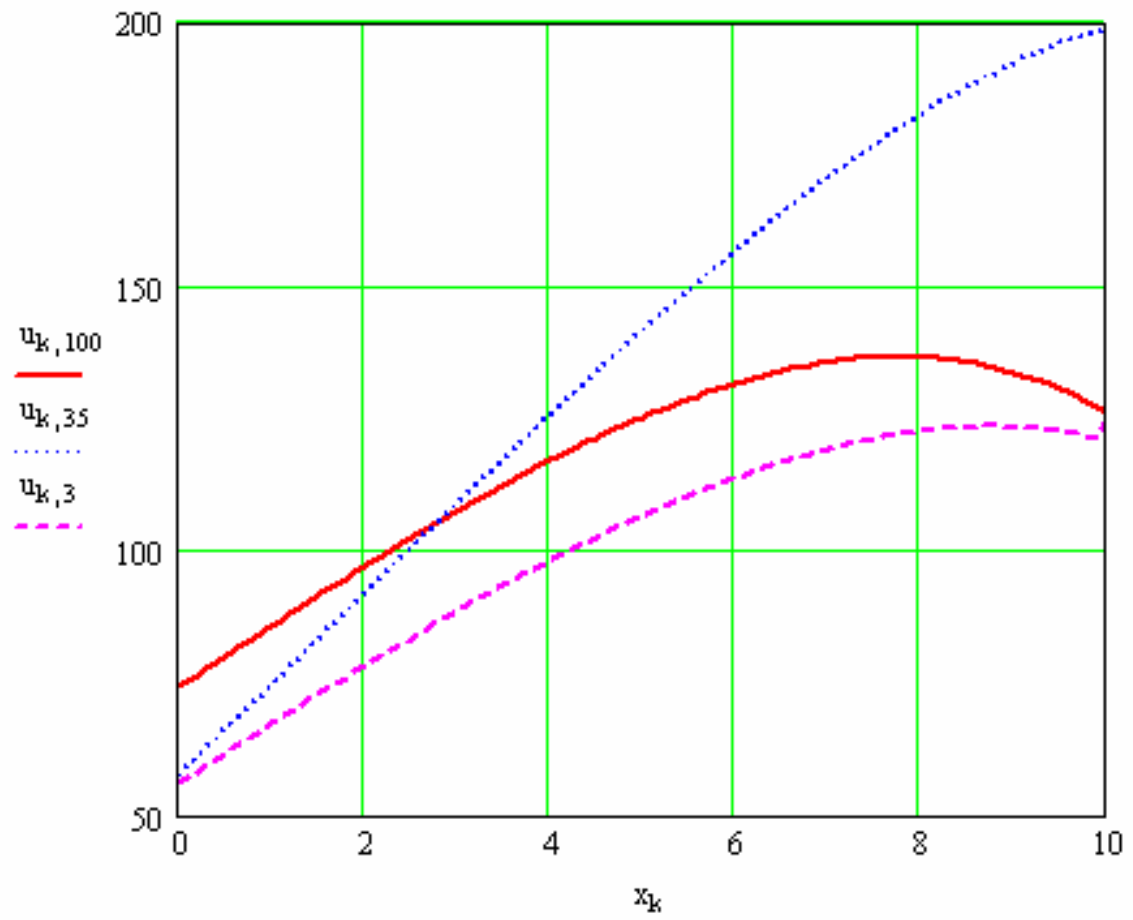

Figure 4. Dependence of temperature on coordinate at time moments.

$$
u\left(x_{k}, t_{100}\right), u\left(x_{k}, t_{35}\right), u\left(x_{k}, t_{3}\right)
$$




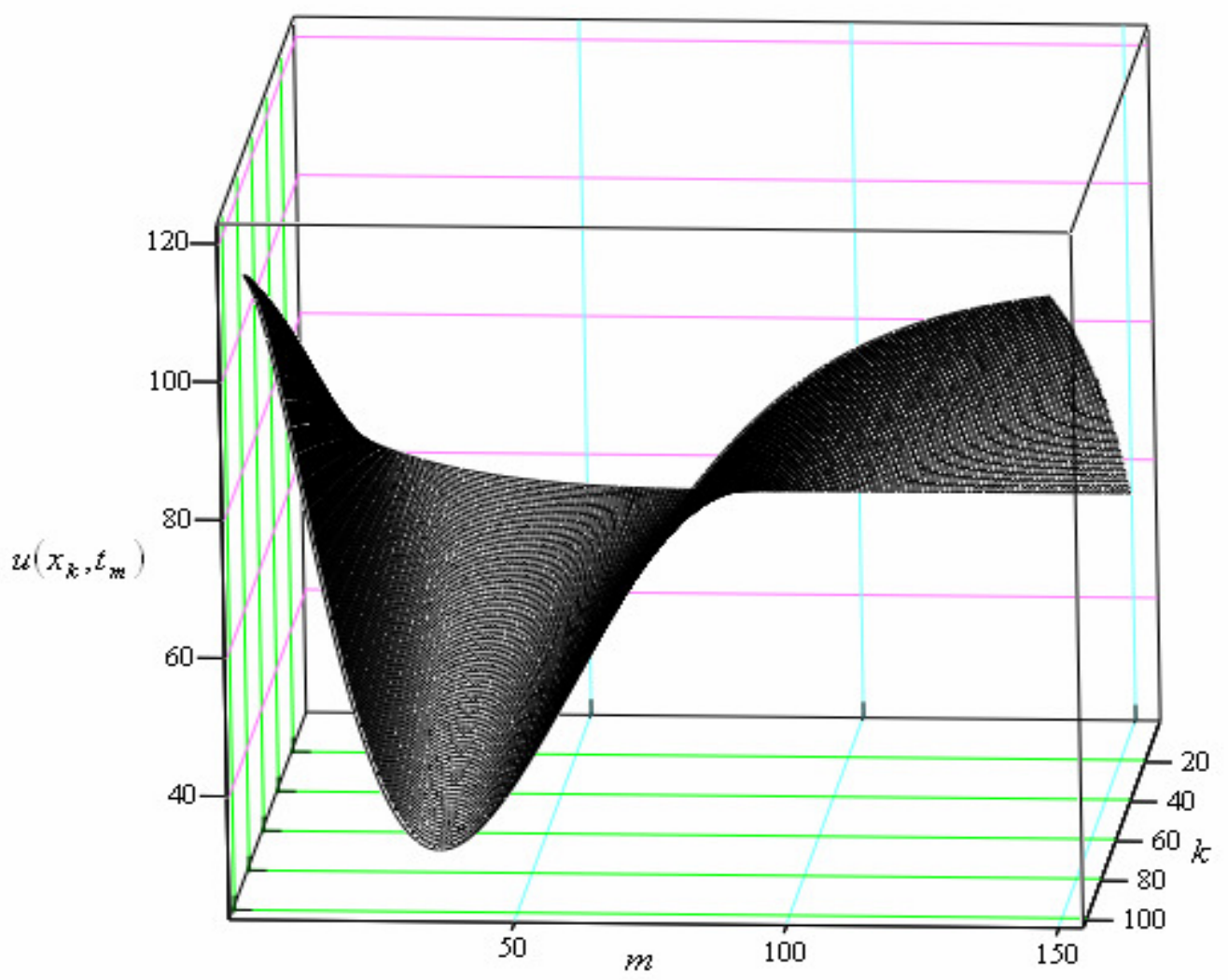

Figure 5. Temperature field $u=u(x, t)$ in the layer.

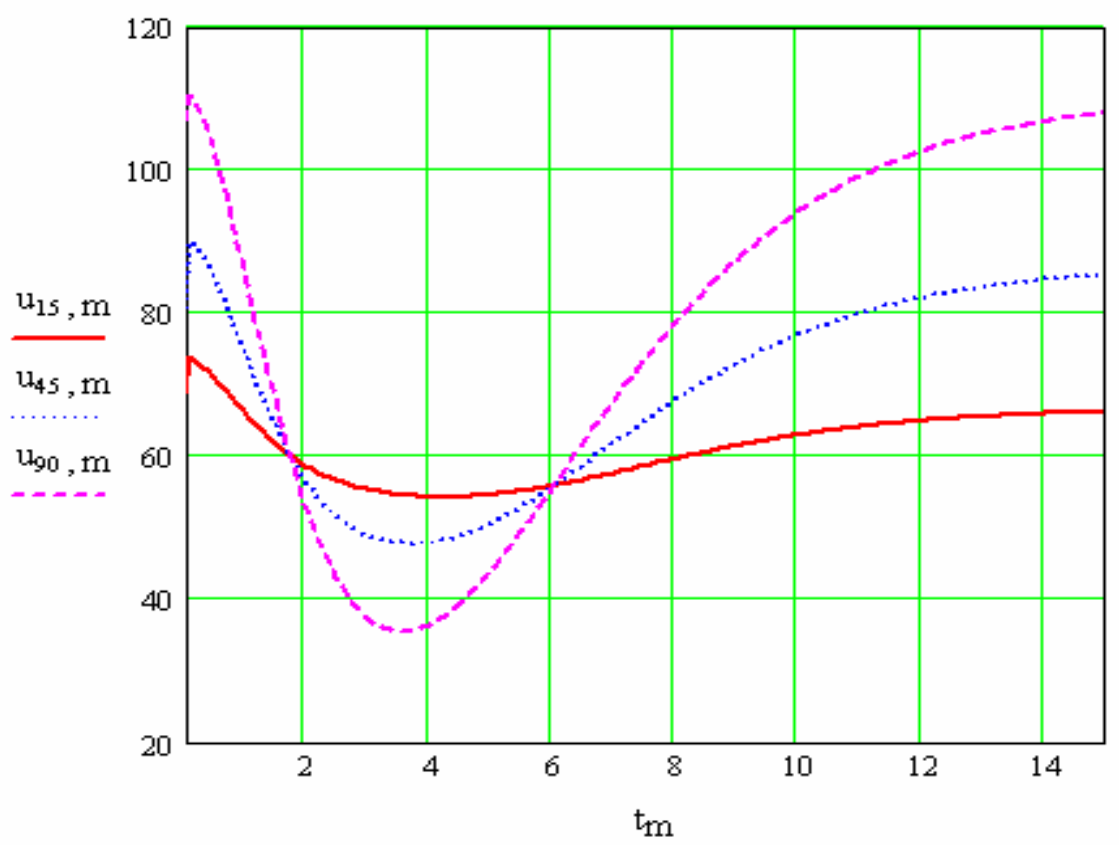

Figure 6. Dependence of temperature on time in the layer sections. 


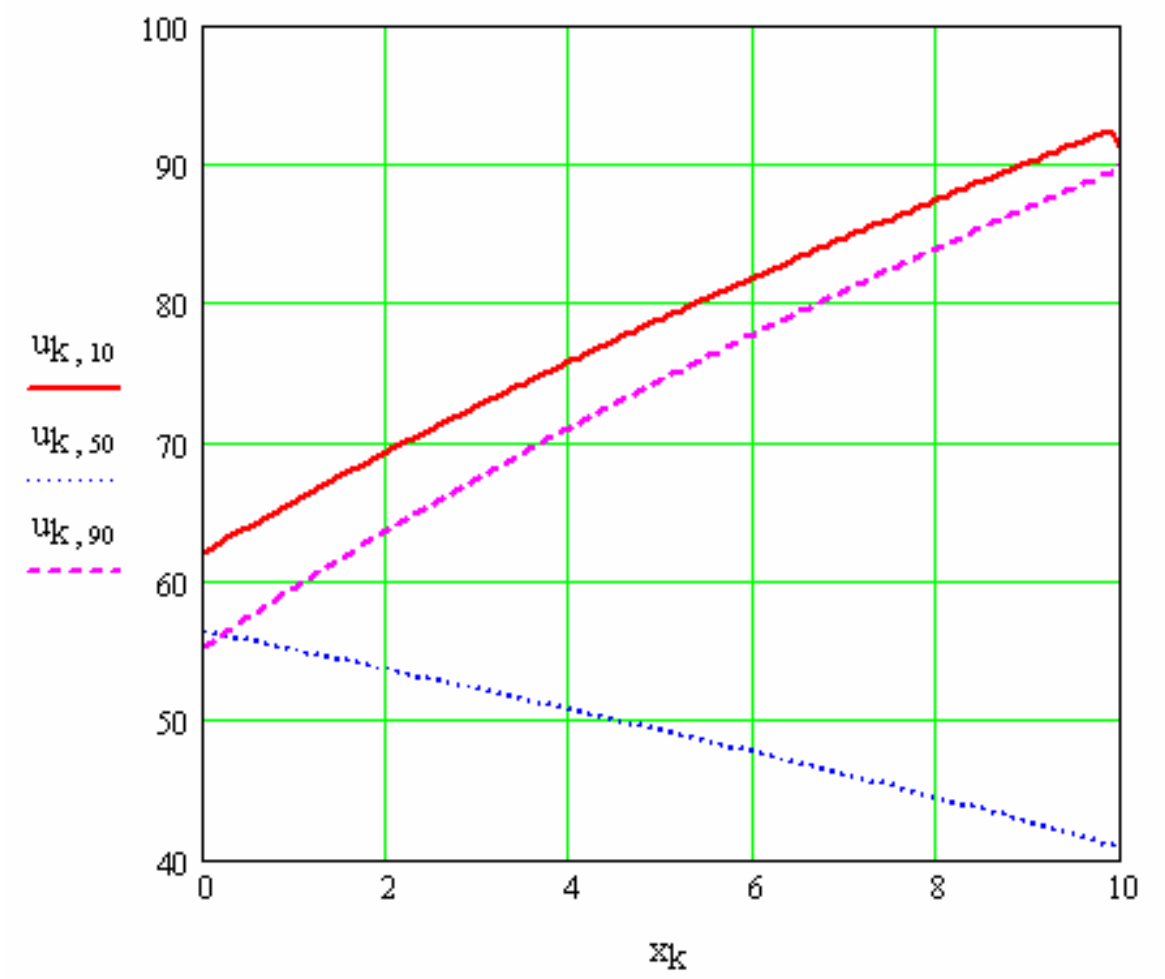

Figure 7. Dependence of temperature on coordinate at time moments. 